\title{
Correlation of the lower limb anatomical shortening with scoliotic deformity of the spine
}

\author{
Viktor Vasilyev ${ }^{1}$, Tamara Begidova ${ }^{1 *}$, Ekaterina Mukina $^{2}$, Oksana Shvachun ${ }^{3}$, Elena \\ Prytkova $^{3}$ \\ ${ }^{1}$ Voronezh State Institute of Physical Training, 394036, Voronezh, Russia \\ ${ }^{2}$ Derzhavin Tambov State University, 392000, Tambov, Russia \\ ${ }^{3}$ Russian State Academy of Justice, 394006, Voronezh, Russia
}

\begin{abstract}
The article deals with the analysis of the correlation between the anatomical shortening of the lower limb and the formation of a scoliotic curve. Despite the fact that the subject matter lacks sufficient research, an attempt is made to consider the data of domestic and foreign scientific studies that shed light on the issue. Within the framework of data analysis in an independent study of a group of 47 patients with shortening of the lower limb combined with vertebral scoliotic deformity, 4 different types of compensatory deformities of the spine were determined. Variants of possible correction were suggested depending on the type and complexity of the deformity. The conducted study stresses the importance of further theoretical and practical development of the subject matter and improvement of therapeutic and preventive measures for scoliosis.
\end{abstract}

\section{Introduction}

Modern society places high demands on specialists in the field of medical and physical rehabilitation for recuperation and preservation of health. These demands are determined by a predominantly sedentary lifestyle, down to and including adynamia, negative effects of environmental factors and low-quality nutrition. Musculoskeletal disorders constitute one of the significant groups of dysfunctions that reduce labour productivity, cause regular inability to work, and sometimes physical disability [1].

Furthermore, scoliotic deformity has an aggressive course and provokes persistent pain symptoms. Whereas the inability to move without pain further stimulates a person to limit motor activity, thus resulting in hypodynamia. Besides, chronization of back pain leads to the loss of economic and everyday independence, ruins the social bonds of a person, causing acute emotional stress.

The visual asymmetry of the body starts from the misalignment of the shoulder girdle level in early stages of the disease. It as scoliosis develops, transforms into a hump in the thoracic region of the spine. It is complemented by the obvious asymmetry of the chest and pelvis. These changes lead to disturbances of vital patterns of respiration, as well as walking and running. The development of muscle imbalances in the form of hypertonia and hypotonia of certain muscle groups, formation of articular restrictions down to contractures,

\footnotetext{
*Corresponding author: begidova@yandex.ru
} 
impaired motor coordination and muscle atrophy are the factors that complement spinal pain symptoms. These factors contribute to pain expansion to the joints of the upper and lower extremities. Even if scoliotic disease stops its development after the closure of growth plates and does not progress further, without corrective efforts the above factors will remain, discomforting the working process and everyday life, decreasing working capacity and cognitive abilities, causing persistent depressive mood and sense of inferiority.

The relevance of this problem is growing in the light of the increased retirement age in Russia. Whereas it is necessary to prolong active ageing.

Scoliotic deformities are a very common kind of the musculoskeletal system diseases. It is worthwhile noting that idiopathic forms of scoliosis were earlier predominant, those with no scientific explanation of their etiology or prediction of deformity development. However, in recent decades there has been an increase in the cases of scoliosis of a structural nature.

The causes of scoliotic deformities of a structural nature include adhesive processes, fibrosis and contractures in the area of the paravertebral muscles, iliopsoas muscle, joints and muscles of the inner side of lower extremities. Analysis of such disorders development biomechanics reveals a general mechanism consisting in the anatomical shortening of the limb causing distortion of the pelvis, disturbing the position of the vertebral column. The studies show that the discrepancy in the length of the lower limbs is recorded in $15-84 \%$ of children and adults, with an average difference of 0.3 to $1.5 \mathrm{~cm}$ [2]. Meanwhile, medical and sport-pedagogical literature hardly ever establishes any correlative interaction of the lower limbs different length and scoliotic deformity. Orthopedic practice completely ignores the asymmetry of the lower limbs as an etiological factor of the scoliotic curve formation.

Attempts of interdisciplinary interaction with specialists, practising in the field of traumatology and orthopedics, for the purpose of diagnosing research subjects often reveal disagreement in the understanding of the scoliotic disease etiology. The replacement of the structural scoliosis notion with the notion of idiopathic scoliosis is presented as the only possible and correct solution followed by the idea of surgical treatment for a progressive scoliotic curve. Noteworthy are the cases of diagnosing scoliotic disease solely on the basis of visual inspection of the lordosis and kyphosis sagittal index changes, or disturbance of the gravity line as a result of one lower limb shortening, or functional asymmetry of the pelvis. Meanwhile, a scoliotic curve is not formed, but the diagnosis arouses fear of physical training in patients. Such examples point out the imperfection of specialist training in certain educational institutions and indicate the necessity of corrections applied to the curriculum of the corresponding disciplines, further fostering the relevance of this study.

The goal of the present research is to analyze the existing studies on the given matter for further establishing of the correlation between the shortening of the lower limb and the formation of a compensatory scoliotic curve with the use of an experimental approach. For this purpose, it is necessary to study the types of compensatory curves and possible ways of their correction.

\section{Materials and Methods}

The study was conducted from September 2019 to September 2020 on the premises of a medical centre "Tri Bogatyrya", Voronezh, Russia.

The study involved 36 women and 11 men aged 16 to 38, living in the city of Voronezh. All research subjects had scoliotic deformities of the vertebral column of 12 to 38 Cobb angle degrees and anatomical shortenings of one lower limb in the range of 0.5 to $2.7 \mathrm{~cm}$.

The study did not involve persons with previous orthopedic or psychosomatic diseases, organic lesions of the CNS or functional shortenings of a lower limb (caused by the valgus 
or varus orientation of the knee joints or feet with equal length of the tibia and the femur). The assessment of scoliotic deformities and detection of different lengths of the legs were carried out with the use of plain X-ray images of the lower extremities and the vertebral column. X-ray images were taken in the functional upright position. A number of objects were additionally used for obtaining anthropometric data: a plumb line, a measuring tape, footrests of different heights.

\section{Results and Discussion}

The discussions of the scientific community keep expanding over the idea of close interdependences between even remotely located biomechanical units. As for the biomechanics of the vertebral column, it is closely associated with the biomechanics of the lower limbs, as the pelvic bones are connected to the sacrum in the sacroiliac joint. The lower limbs and the pelvis transmit gravity and support reaction forces to the vertebral column in the pattern of walking. Many authors agree that there is a direct correlation between unilateral shortening of the shin and thigh bones and impaired spinal biomechanics $[7,9,11,12,13]$.

The study of schoolchildren showed that long-term asymmetric load while the growth plates are still open can serve as a trigger for the development of scoliotic deformities [2, 4]. A sharp increase in scoliotic deformities in recent decades is explained in the study of children. They developed scoliotic disease having muscular hypotonia and a discrepancy in the leg length of $0.3-0.5 \mathrm{~cm}[5,6]$.

A team of authors have been working on a theory of the scoliotic deformity development with different length of the lower limbs [2, 7-10]. According to the theory, the anatomical shortening of the shin and/or thigh bones on one side leads to an oblique position of the pelvis, followed by the displacement of the sacrum and consequently the entire vertebral column. This is how the vertebral column forms the primary curve in the frontal plane. Therewith, the head tilts to the side making the functioning of the vestibular apparatus and the visual analyzer ineffective. So the head reflexively takes a proper vertical position. Whereas the vertebral column compensatorily forms a counter-curve, opposite to the primary one, or increases the radius of the primary curve. Such deviation initially exists only in the frontal plane, although the load on the vertebral bodies and intervertebral discs is distributed unequally. In case of pelvic asymmetry the pattern of walking is performed ineffectively, accompanied by disturbance of the coxofemoral joint rotation on the side of the longer limb, which leads to the compensatory rotation in the vertebral column, blockage of the facet joints and rotation of the vertebral bodies. It is important that the earlier the conservative treatment is started, the more effective it is. Derotation exercises are the most difficult to perform, and are not always effective due to the rigidity of the thorax $[6,9]$.

Theoretical insights find evidence in clinical practice. Thus, the team of authors [5] discovered that correction of the lower limb shortening with an orthopedic insole had straightened the scoliotic curve in $78 \%$ of the subjects, while $22 \%$ of the subjects had shown no reduction of scoliotic deformity. Some subjects had even presented with the opposite effect in the form of the curve progression, emergence of expressed asymmetry and appearance of a new curve. Such response to correction explicitly indicates that disorders in the vertebral column are persistent and correction of the leg length alone can sometimes be not enough for effective therapy.

In addition to the anatomical shortening of the lower limb leading to disturbance of the normal biomechanics of the pelvic region called "pelvic obliquity", there is also a functional type of the lower limb shortening. The functional shortening is characterized by equal anatomical length of the tibia and femur. However, the muscle imbalance of the coxofemoral joint flexors and extensors, specifically shortening of the flexor muscles on 
one side and shortening of the extensor muscles on the other side, leads to a static and dynamic disorder called "oblique pelvic tilt".

Regarding the anatomical shortening when all 3 anatomical landmarks of the pelvis (anterior superior iliac spine, posterior superior iliac spine, iliac crests) are located higher or lower compared to the contralateral side, the following peculiarity can be revealed: the anatomical landmarks of the pelvis are displaced more asymmetrically in the functional shortening. For example, the posterior superior iliac spine and the right iliac crest are higher compared to the same landmarks on the contralateral side. While the anterior superior iliac spine is conversely lower on the right than on the left [6].

Such disturbance of the statics and dynamics of the pelvis entails displacement and rotation of the sacrum and then the entire vertebral column. Thus, functional asymmetry is also a precursor for the development of scoliotic disease.

The equality of all control points height on the right and on the left can be the evidence of the pelvis physiological position and equal length of the lower limbs. However, if the control points on one side are higher or lower than on the other side, the shortening of one leg can be assumed. This assumption can be confirmed by measurements of the lower limbs, possible to be conducted in two different ways.

The first way is to measure from the anterior superior iliac spine to the medial condyle of the tibia. The second way is to measure the shin and the thigh separately: the thigh from the greater trochanter to the distal lateral condyle of the femur, and the shin - from the proximal lateral condyle of the tibia to the distal condyle. Such measurements allow to exclude functional shortenings of the lower limb, such as valgus (x-position) or varus (oposition) deformities of the legs or flattening of the foot arch. However, the final conclusion on the shortening of the tibia or femur is made after reading an X-ray image.

When selecting complexes of exercises having specified the shortening of the limb, it is necessary to determine whether the line of the primary scoliotic curve corresponds with the pelvic asymmetry. If the leading curve is bent to the side of the shortened limb, and the tilt of the sacrum is co-directed with the tilt of the pelvis, there is a very high probability of a direct correlation between the anatomical shortening of the lower limb and the development of the scoliotic curve. In such case the effect of special scoliosis-correction exercises will be much better, provided that the position of the pelvis is corrected by equalizing the length of the limbs. This can be achieved by wearing orthopedic shoes, high insoles or heel pads compensating for the limb shortening. The effect is determined by the restoration of gluteal muscle tone. It is possible only when the pelvis is aligned.

Alignment of the pelvis improves functioning of the deep back muscles: multifidus muscle, erector spinae muscle, quadratus lumborum muscle, psoas major muscle. Improving muscle tone provides better stabilization of the vertebral column, aligning its position in the frontal and sagittal planes [2].

The study, conducted on the premises of a medical centre "Tri Bogatyrya" in Voronezh, involved 36 women and 11 men aged 16 to 38 years with scoliotic disease of I-III degree with Cobb angle of 12-38 degrees and the anatomical shortening of one lower limb in the range of 0.5 to $2.7 \mathrm{~cm}$.

The study did not involve persons with functional shortenings of the lower limbs (caused by the valgus or varus orientation of the knee joints or feet with equal length of the tibia and the femur, or muscle imbalance of the coxofemoral joint flexors and extensors), previous orthopedic or psychosomatic diseases, or organic lesions of the CNS. The study of the lower limbs shortening, pelvic asymmetries and scoliotic deformities was conducted with the use of plain X-ray images of the lower extremities and the vertebral column, supplemented with visual diagnostics of the anatomical landmarks of the pelvis.

As a result of the diagnostics, the research subjects were divided into 4 groups depending on the type of deformity of the spine and the pelvis. 
Group 1 - spinal deformity with a curve laterally aligned with the pelvic tilt (the apex of the curve is co-oriented with the shortened limb) - involved 14 women and 4 men with the anatomical shortening of the lower limb of 0.7 to $2.1 \mathrm{~cm}$ and a scoliotic curve of $12-22$ Cobb angle degrees. In 6 women and 1 man, the scoliotic curve was not compensated, that is, the level of the 7th cervical vertebra did not coincide with the level of the sacrum along the plumb line. It indicated that the scoliotic curve was still in the process of formation. This group with the direct association of the pelvic tilt with the scoliotic curve showed the most effective results of correction. All subjects were consulted by an orthopedic doctor and received correction of the anatomical shortening by fitting orthopedic insoles or heel pad.

The correction resulted in reduction of scoliotic deformity in all subjects by 8 degrees on the average; in addition supporting physical exercises were recommended with X-ray control after 6 months.

Group 2 - spinal deformity with a leading lumbar curve laterally aligned with the pelvic tilt and a formed thoracic counter-curve - involved 12 women and 2 men with the anatomical shortening of the lower limb of 0.5 to $2.7 \mathrm{~cm}$ and the Cobb angle of the leading curve of 16-38 degrees. All subjects had compensated scoliotic deformity. The correction resulted in reduction of the leading curve scoliotic deformity in all subjects by 12 degrees on the average. Although in some cases, the thoracic counter-curve showed a tendency to increase by 5 degrees on the average. The subjects were recommended with exercises to improve the mobility of the thoracic spine, asymmetric breathing techniques by the Katharina Schroth's system and exercises for active self-correction by the SEAS system (Scientific Exercise Approach to Scoliosis, Italy).

Group 3 - spinal deformity with a curve laterally misaligned with the pelvic tilt (the apex of the curve is disoriented with the shortened limb) - involved 6 women and 5 men with the anatomical shortening of the lower limb of 0.7-1.2 cm and the Cobb angle of 15-21 degrees. Correction of the lower limb length was not recommended for any of the subjects, since it resulted in the compensatory increase of the scoliotic curve in all cases. The subjects were recommended with the complexes of therapeutic exercises based on the systems by Katharina Schroth and the SEAS (Scientific Exercise Approach to Scoliosis, Italy) under the instructor's guidance, followed by X-ray control after 9-12 months to make a decision on the correction of the lower limb shortening.

Group 4 - deviations of the spine axis without the formation of a scoliotic curve; the imbalance of the trunk, laterally aligned with the pelvic tilt by the type of "The Leaning Tower of Pisa" - involved 4 women with the shortening of the lower limb of 0.6 to $1.1 \mathrm{~cm}$. The scoliotic curve was not formed; however, the plumb line diagnosis revealed deviation of the 7th cervical vertebra from the sacrum by $1.5-2.5 \mathrm{~cm}$. It creates preconditions for scoliosis formation and allows including these cases in our study. After a consultation with an orthopedic doctor, the decision was made to correct the shortening of the lower limb by $50-70 \%$. They led to an improvement in the alignment of the pelvis and reduction of progressing spinal deformity with a deviation of $0.7-1.2 \mathrm{~cm}$. A higher degree of correction caused the formation of a scoliotic curve. The subjects were indicated with exercises to improve the mobility of the thoracic spine, followed by X-ray control during 9-12 months.

Group 5, consisting of 1 woman and 3 men with pelvic tilt due to the shortening of the lower limb, happened to be beyond the scope of the study. The plumb line test, supplemented by the Adams functional test and a plain X-ray image of the vertebral column showed that the spine maintained a strictly vertical position without distortions in the frontal plane. Such unusual situation with no compensatory spine deformity caused by pelvic tilt can be explained by high flexibility of the paravertebral soft tissues and high mobility of the spine itself, which determined its optimal position. 
Apart from the visual difference in the height of the iliac crests, there were no other signs of scoliotic disease, X-ray imaging did not confirm the presence of scoliotic deformity; therefore, the decision was made not to include these patients in the study. Considering the absence of clinical symptoms or functional disorders, the authors did not see it necessary to correct such deformities.

The results of the study with the corrective measures applied in the course of it and with a positive therapeutic prognosis show that the degree of scoliotic deformities has decreased by 10 degrees on the average. Each subject from both groups of patients: those who received correction of the shortening of the lower limb and those under "watchful waiting", was recommended with individually selected exercises.

The first and fourth groups did exercises aimed at improving the mobility of the spine. The second and third groups in addition to exercises improving the mobility were recommended with exercises aimed at specific correction of scoliotic deformity. It showed their effectiveness.

To summarize the results of the study, patients of all groups mentioned reduction of pain in the back and neck from 6-7 points to 1-2 points according to the visual analogue scale. Moreover, the study revealed a decrease in fatigue, an increase in working ability and normalization of blood pressure.

\section{Conclusions}

- The anatomical shortening of the lower limb causing pelvic asymmetry is an important factor influencing the development of scoliotic deformity;

- Correction of the anatomical shortening using orthopedic insoles has an expressed corrective effect, reducing the size of the scoliotic curve;

- In some cases, the anatomical shortening of the lower limb does not lead to the formation of a scoliotic curve;

- Correction of the anatomical shortening is more effective in combination with specially selected complexes of therapeutic exercises;

- A combination of orthopedic correction measures with the use of exercise complexes leads to reduction of pain syndrome and improvement of the quality of life;

\section{Acknowledgements}

To my scientific advisor and ideological inspirer, professor, candidate of pedagogical sciences Tamara Pavlovna Begidova for support and motivation.

\section{References}

1. V.E. Belenkiy, N.N. Priorov J. of Traumat. \& Orthop. 1, 47-51(1994)

2. V.A. Vasiliev, Modern Vectors of Appl. Res. in the Field of Phys. Cult. \& Sports, 120 $122(2020)$

3. A.V. Gladkov, Spine surgery 2, 53-57 (2004)

4. A.V.Gubin, P.E. Lozovskiy, S.S. Peredereev, Topical Issues of Pediatric Traumat. \& Orthop., 93-94 (2005)

5. N.A. Zinchuk, N.V. Yermolina, A.V. Dorontsev, Pedagogico-phsycological and medico-biological problems of physical culture and sports, 12(2), 92-97 (2017)

6. V.A. Lanshakov, VII Congr. of Traumat.-Orthop. of Russia, 148-149 (2002)

7. D.B. Simkin, Man and his health 2, 186 (1996)

8. K. Bagnall, Scoliosis 3, 1022-1028 (2008) https://doi.org/10.1186/1748-7161-3-5 
9. R. G. Burwell, Acta Orthopaedica Belgica 3, 247-260 (2006) https://doi.org/10.1186/s13013-016-0105-8

10. A. Manganiello, Radiol. Med. 71(5), 298-302 (1985) https://doi.org/10.1186/s40945015-0001-1

11. D. L. Specht, J. Manipul. Physiol. Ther. 14(6), 368-375 (1991) DOI: 10.3233/978-161499-067-3-146

12. T. Yrjonen, J. Spinal Disord. 5(4), 443-447 (1992) DOI: 10.1097/00002517199212000-00009

13. M. Walsh, J. Pediatr Orthop. 4, 537-541 (2006) http://dx.doi.org/10.1097/BPO.0b013e3181558b8d 